\title{
PIN diamond detector development for LYRA, the solar VUV radiometer on board PROBA II
}

\author{
A. BenMoussa ${ }^{*}, 1$, U. Schühle ${ }^{2}$, K. Haenen ${ }^{3}$, M. Nesládek ${ }^{3,4}$, S. Koizumi ${ }^{5}$, \\ and J.-F. Hochedez ${ }^{1}$ \\ ${ }^{1}$ Royal Observatory of Belgium, Circular Avenue 3, 1180 Brussels, Belgium \\ 2 Max Planck Institut für Sonnensystemforschung - 37191 Katlenburg-Lindau - Germany \\ ${ }^{3}$ Institute for Materials Research (IMO), Limburgs Universitair Centrum, Wetenschapspark 1, \\ 3590 Diepenbeek, Belgium \\ ${ }^{4}$ Division IMOMEC, IMEC vzw, Wetenschapspark 1, 3590 Diepenbeek, Belgium \\ 5 Advanced Materials Laboratory, National Institute for Materials Science (NIMS), 1-1 Namiki, \\ Tsukuba 305-0044, Japan.
}

Received 1 July 2004, accepted 31 July 2004

Published online 7 September 2004

PACS 85.60.Bt, 85.60.Gz

LYRA (the LYman-alpha RAdiometer onboard PROBA-2) will use diamond detectors for the first time in space solar physics. A set of measurement campaigns was designed to obtain the EUV-to-VIS characterization of the diamond detectors and knowledge of their modes of operation. The outputs of this campaign provide the reference data needed for the optimization of the detectors, and for the LYRA channels definition. The measurements of responsivity in EUV and VUV spectral ranges have been carried out by the Physkalisch-Technische Bundesanstalt (PTB) in Germany at the electron storage ring BESSY II. The detectors spectral responsivity measurements $R(\lambda)$ was done for the following wavelength ranges: 40 to $80 \mathrm{~nm}, 80$ to $120 \mathrm{~nm}$ and 120 to $240 \mathrm{~nm}$. The longer wavelength range from $210 \mathrm{~nm}$ to $827 \mathrm{~nm}$ was measured with the monochromatic light by using a Xe-lamp at IMO-IMOMEC. The diodes exhibit high photoresponse in the deep UV region and over five orders of magnitude in discrimination between deep UV $(200 \mathrm{~nm})$ and visible light $(550 \mathrm{~nm})$.

() 2004 WILEY-VCH Verlag GmbH \& Co. KGaA, Weinheim

\section{Introduction}

LYRA (LYman-alpha RAdiometer) [1] is the solar VUV radiometer that will embark in 2006 onboard PROBA-2, a technologically oriented ESA micro-mission. It is a compact instrument that accompanies SWAP, a solar EUV imaging telescope. LYRA will be complementary to instruments that have flown or are presently flying on space missions monitoring the solar UV irradiance. LYRA will monitor the solar irradiance in four selected UV passbands. The channels have been chosen for their relevance to Aeronomy, Space Weather and Solar Physics [2, 3]: 1/Lyman-alpha (121.6 nm), 2/the 200-220 nm Herzberg continuum range (interference filters for the two former passbands), 3/Al filter channel (17-70 nm) covering He II- $30.4 \mathrm{~nm}, 4 / \mathrm{XUV} \mathrm{Zr}$ channel $(1-20 \mathrm{~nm})$, where solar variability is highest. This will make LYRA a unique flare monitor and an alternative to GOES-N [4] operating at even higher energies. All channels require a robust radiometric calibration (accuracy, stability) to convert digital units into photon fluxes at any time of the mission, and under any condition (flatfield, linearity over two decades). LYRA will benefit from diamond detectors: it will be the first space assessment of a pioneering UV detector program $[5,6]$. Diamond, a wide bandgap material, makes the sensors "solar-blind", which allows sup-

\footnotetext{
* Corresponding author: e-mail: ali.benmoussa@oma.be, Phone: +32 2373 0276, Fax: +32 23730224
} 
pressing some of the usual filters, that block the unwanted visible, but attenuate seriously the desired UV radiation. Therefore, their removal enhances the effective area, increases the signal to noise, the cadence, or an optimal trade-off of these two. A redundancy strategy maximizes the precision and the stability of the measurements. The instrument and the software are such that LYRA will be an innovative solar monitoring tool for operational space weather nowcasting and research.

\section{$2 \quad 1^{\text {st }}$ pre-flight detector calibration campaign}

The first of the LYRA synchrotron campaigns was performed using the Normal Incidence (NI) beamline of the Physikalisch-Technische Bundesanstalt (PTB) Laboratory at the Berliner ElektronenspeicherungGesellschaft für Synchrotronstrahlung (BESSY II) over the spectral range from $40 \mathrm{~nm}$ to $240 \mathrm{~nm}$. The longer wavelength range from $210 \mathrm{~nm}$ to $827 \mathrm{~nm}$ was measured at IMO-IMOMEC using a monochromatized Xe-lamp. The detector calibration facility at BESSY II provides a synchrotron beam, monochomatized by a normal-incidence monochromator (with a spectral resolution of $0.1 \mathrm{~nm}$ ). To cover a wide spectral range from $40 \mathrm{~nm}$ to $240 \mathrm{~nm}$, different filters and beamline mirrors must be used to suppress higher order contributions to the selected bandwidth. This divides the entire range into three sub-ranges: $40 \mathrm{~nm}-80 \mathrm{~nm}, 80 \mathrm{~nm}-120 \mathrm{~nm}, 120 \mathrm{~nm}-240 \mathrm{~nm}$. Between these ranges, filters and mirrors will be changed so that a spectral cleanliness is guaranteed to the level that higher order contributions are negligible. The detector calibration chamber at the end of the beamline is an ultrahigh vacuum chamber with manipulation stages, which allows the sample holder to be moved along three axes relative to the beam axis. The manipulation stages allow to raster the sample area and to switch between test and reference detectors. The spot size of the beam at the location of the samples is approximately $1 \times 2 \mathrm{~mm}$. This depends slightly on the chosen wavelength. The radiometric uncertainty of the measurements was estimated to be better than $10 \%$ in absolute terms.

\subsection{Description of the diamond photodetector}

A diamond photodetector has been investigated (Fig. 1). The sample was designated PIN2 in the program and also in the following text. The PIN2 sample is a P-i-N diode. The homoepitaxial diamond layers of the detector were deposited using two ULVAC stainless steel chamber plasma-enhanced microwave deposition reactors. After a careful selection of a $\{111\}$-oriented $2 \times 2 \times 0.5 \mathrm{~mm}^{3} \mathrm{HPHT}$ Ib substrate from Sumitomo, followed by a chemical oxidation, an epitaxial p-type B-doped layer was deposited using a mixture of methane, hydrogen and trimethylboron (TMB). Typical process parameters are a pressure of 100 Torr, a substrate temperature around $900-950{ }^{\circ} \mathrm{C}$ and a microwave power of about $400 \mathrm{~W}$. A low-concentration methane-to-hydrogen mixture of $0.05 \%$ is used, while the TMB-to-methane ratio was varied between 100 and $4000 \mathrm{ppm}$. After moving the sample into the second deposition system, the intrinsic $\left(\mathrm{H}_{2}\right.$ and $\left.\mathrm{CH}_{4}\right)$ and $\mathrm{P}$-doped $\left(\mathrm{H}_{2}, \mathrm{CH}_{4}\right.$ and phosphine) layers are added. These layers are grown at a slightly higher microwave power $(700 \mathrm{~W})$ and lower substrate temperature $\left(870-900{ }^{\circ} \mathrm{C}\right)$, while the $\mathrm{PH}_{3} / \mathrm{CH}_{4}$ ratio is about $5000 \mathrm{ppm}$ for the n-type layer.

Following the growth procedure, several circular ohmic Ti/AU contacts $(\varnothing 150 \mu \mathrm{m})$ are evaporated onto the P-doped surface, followed by a thin Al layer. This layer, which is chemically removed after

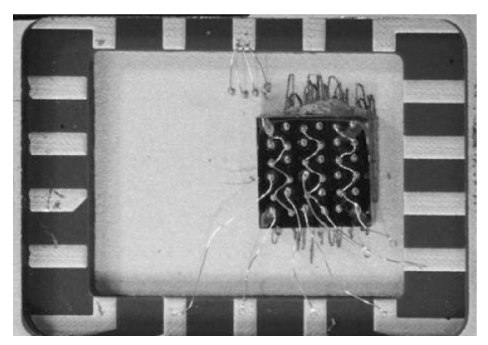

Fig. 1 Photograph of the PIN2 diode. 
wards, acts as a mask during the reactive ion etching, necessary to reach the B-doped layer. As a last step, ohmic contacts are also deposited on the p-type layer. For a more detailed description on the growth of diamond pn-junctions, we refer to Refs. [7, 8].

\section{Experimental results}

The spectral responsivity was measured in comparison with a reference detector, mounted on the same sample holder: A PtSi-Si Schottky photodiode with an absolute calibration made at the electron storage ring BESSY II [9]. The responsivity of this reference diode is shown in Fig. 2.

The sample had a square size of about $2 \times 2 \mathrm{~mm}$ although flight model detectors will be $5 \mathrm{~mm}$ diameter. This made it necessary that an aperture stop had to be specially built, in order to avoid spurious signal from irradiation of the surrounding contacts. Before the measurement of responsivity, the time to reach a stable signal was measured and the stability of the signal and dark signal was checked.

\subsection{Time response and signal stability of the device}

The stability of the photo-current was investigated first by a scan of the signal versus time, in order to characterise the time response of the sample. The signal stability may be a concern for the timing of the measurements. Therefore, the time response has been determined by recording the signal and switching the light beam on and off. The PIN2 sample response was measured at $55 \mathrm{~nm}$ (Fig. 3) without bias voltage (photovoltaic mode). The data shown in this figure are corrected for the small decline (of $1 \%$ ) of the current of the synchrotron storage ring during the time period of each measurement.

The PIN2 sample shows a fast response to the signal change and an exact return to the dark-signal after irradiation. A small decline of the signal during the 160 seconds of irradiation remains to be explained and may be a concern for long-term stability. As a derived result, the $\mathrm{S} / \mathrm{N}$ can be measured at this wavelength. It shows a factor $10^{3}$ between noise and signal.

\subsection{Responsivity $R(\lambda)$}

The responsivity measurement $R(\lambda)$ consists of a full wavelength scan with the reference diode, the same scan with the test sample, another scan with the reference diode, and three raster scans of the test sample at three different wavelengths. Individual measurements were repeated to check stability and reproducibility. The absolute spectral responsivity curves of PIN2 sample is shown in Fig. 4. Sample PIN2 showed a very good response above the threshold between $220 \mathrm{~nm}$ and $180 \mathrm{~nm}$, with a maximum of $10 \mathrm{~mA} / \mathrm{W}$ at $190 \mathrm{~nm}$. Below $180 \mathrm{~nm}$, the responsivity decreased.

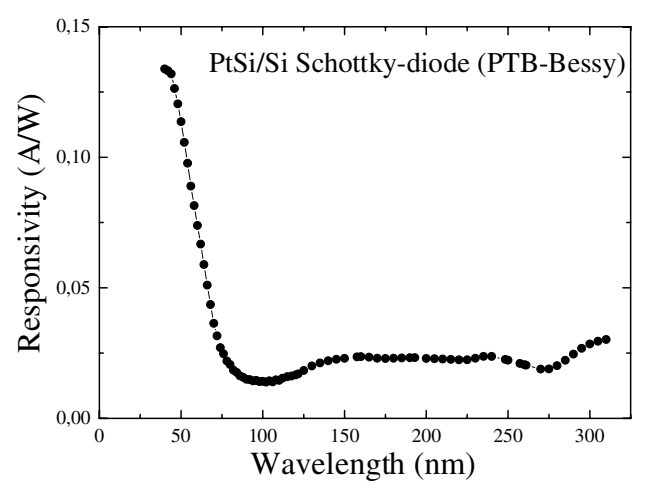

Fig. 2 Responsivity of the calibrated $\mathrm{PtSi}-\mathrm{Si}$ Schottky photodiode.

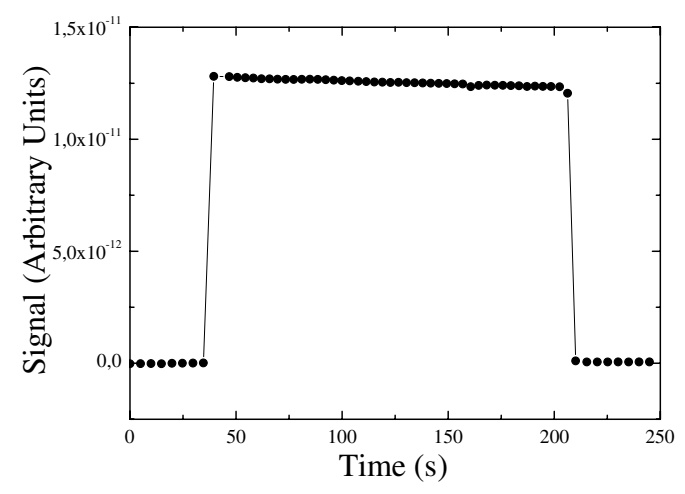

Fig. 3 Time response characterisation of PIN2 sample at $55 \mathrm{~nm}$. 


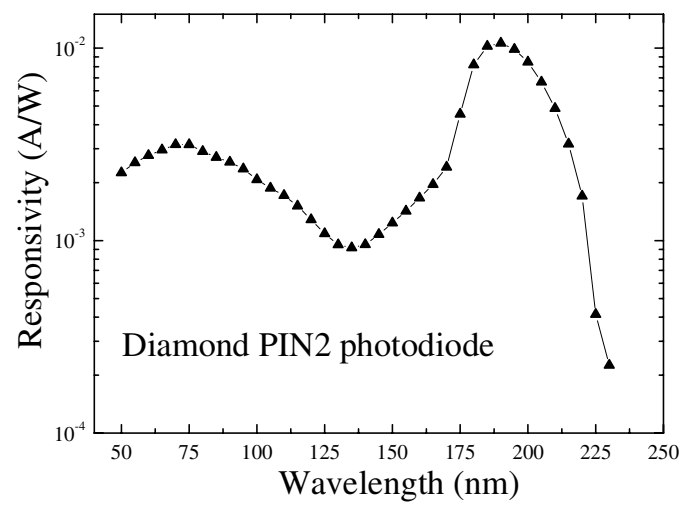

Fig. 4 Absolute spectral response (in A/W) of PIN2 sample between $50 \mathrm{~nm}$ and $230 \mathrm{~nm}$.

The reason could not be identified. Below $120 \mathrm{~nm}$ a maximum was found at $70 \mathrm{~nm}$. The contribution of the photo-emission current [10] to the total signal (internal photo-current) of the photodetector sample has been carried out with PIN2 sample between $110 \mathrm{~nm}$ and $180 \mathrm{~nm}$. The measurement with one and the other side grounded gave the same signal. Thus it was concluded that there is either no photo-emission contribution to the signal in this wavelength region or the emission from the top and bottom layers are equal (the latter can be ruled out by the device geometry).

\section{Solar-blindness tests $(210-827 \mathrm{~nm})$}

A schematic representation of the set-up used to measure the photocurrent is shown in Fig. 5. The sample holder (1) consists of a circular metal holder with two BNC connectors and a Teflon disc inside. The monochromatic light is generated by using a Xe-lamp, a monochromator and some appropriate filters (For the low energy region the Xe-lamp is replaced by a halogen-lamp (3)). For the ultraviolet (UV) region a water cooled UV-enhanced xenon lamp (2) is used.

By means of a flat (4) and concave (5) mirror the light beam is focussed onto the entrance slit of the monochromator (7). The intensity of the light is modulated by a mechanical chopper (6) placed in front of the entrance slit. This enables the use of lock-in techniques for the photocurrent detection, which is beneficial for the $\mathrm{S} / \mathrm{N}$ ratio. Higher order diffraction wavelengths are an unwanted by-product in the output beam. Therefore a filter wheel (8) with 6 different positions is placed directly behind the output slit. A spectrally independent $\mathrm{CaF}_{2}$ beamsplitter (9) directs about $10 \%$ of the light towards a pyrodetector (10) with a uniform response in the wavelength interval under consideration. The majority of the light $(90 \%)$ is transmitted

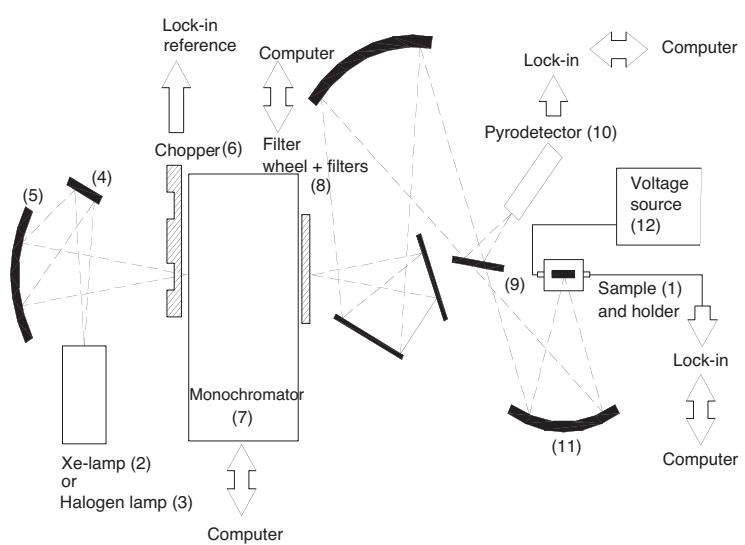

Fig. 5 Schematic representation of the solar blindness tests at IMO-IMOMEC. 


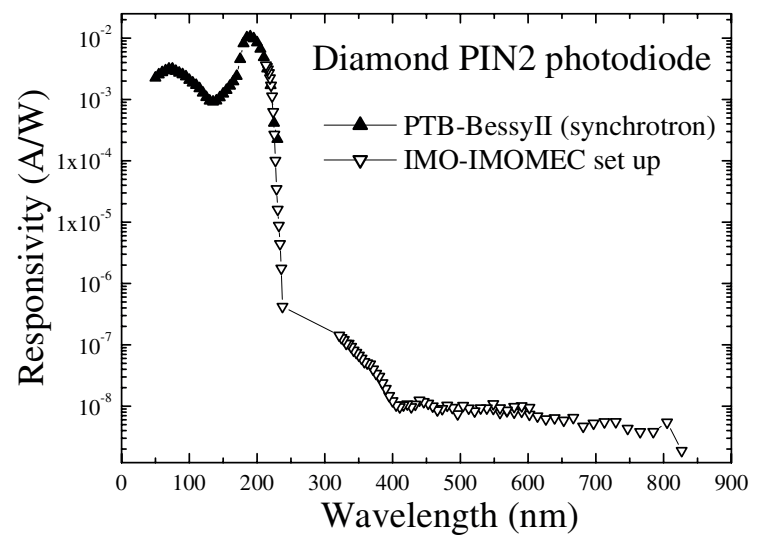

Fig. 6 Measured spectral responsivity (in A/W) of PIN2 sample (50-827 nm).

by the beamsplitter and, via a concave mirror (11), directed onto the sample surface near the gap between the contact structures. These contacts are connected to an appropriate voltage source (12).

The spectral responsivity of PIN2 sample measured in this wavelength range is shown in Fig. 6.

\section{Conclusion - Future campaigns}

Diamond photodetectors (PIN) with an optically active surface area of $4 \mathrm{~mm}^{2}$ have been fabricated. The characteristics of diodes show the sharp diamond band edge around 200-220 nm and exhibit deep UV $(200 \mathrm{~nm})$ and visible light $(550 \mathrm{~nm})$ discrimination over five orders of magnitude. We anticipate still higher ratio in the future due to thinning techniques and improvement of the crystal quality. Improvements in the performance characteristics of our diamond photodetectors is a goal for the LYRA mission. In the next calibration campaign (June 2004), the next generation of diamond detectors $\left(\varnothing 5 \mathrm{~mm}^{2}\right)$ will be measured over a wider spectral range, including the EUV and XUV (grazing incident 1-32 nm) regime.

Acknowledgements The authors wish to thank the Belgium Science Policy (BELSPO) for their financial support. The measurements at PTB/BESSY were made possible by a collaboration between the Max-Planck-Institut für Sonnensystemforschung and the Physikalisch-Technische Bundesanstalt. We gratefully acknowledge the help of M. Richter, U. Kroth, and A. Gottwald of PTB. KH is a Postdoctoral Fellow of the Fund for Scientific Research - Flanders (Belgium) (F.W.O.-Vlaanderen).

\section{References}

[1] J.-F. Hochedez, W. K. Schmutz, M. Nesladek, S. Koller, D. Berghmans, A. Ben Moussa, J. H. Lecat, J. M. Defise, Y. Stockman, L. Wauters, B. Nicula, U. Schühle, D. Gillotay, M. Kretzschmar, H. Roth, E. Rozanov, C. Wehrli, I. Ruedi, R. Van der Linden, A. Zhukov, F. Clette, M. d'Olieslaeger, J. Roggen, and P. Rochus, LYRA: the Solar VUV radiometer on-board PROBA II. ESA Space Weather Workshop: Developing a European Space Weather Service Network SWENET: Space Weather European Network, ESTEC, Noordwijk (3-5 November 2003). http://lyra-swap.oma.be/.

[2] E. Rozanov, T. Egorova, C. Fröhlich, M. Haberreiter, T. Peter, and W. Schmutz, Estimation of the ozone and temperature sensitivity to the variation of spectral solar flux. In: A. Wilson (ed.) SOHO 11 Symposium: From Solar Min to Max: Half a Solar Cycle with SOHO, ESA SP-508, ESA Publications Division, (Noordwijk, The Netherlands 2002) pp. 181.

[3] G. Kockarts, Aeronomy, a 20th Century emergent science: the role of solar Lyman series: Annales Geophysicae 20, 585-598 (2002).

[4] L. Shing, R. A. Stern, P. Catura, and M. Morrison, CCD development and characterization for the GOES N and O Solar X-ray Imager. In Proc. SPIE 3765, 299-309 (1999), EUV, X-Ray, and Gamma-Ray Instrumentation for Astronomy X.

[5] J.-F. Hochedez, U. Schühle, J. L. Pau, J. Alvarez, O. Hainaut, T. Appourchaux, D. Auret, A. Belky, P. Bergonzo, M. C. Castex, A. Deneuville, P. Dhez, B. Fleck, K. Haenen, M. Idir, J. P. Kleider, E. Lefeuvre, P. Lemaire, 
E. Monroy, P. Muret, E. Munoz, M. Nesladek, F. Omnes, E. Pace, A. Peacock, and C. Van Hoof, New UV detectors for solar observations, in: Innovative Telescopes and Instrumentation for Solar Astrophysics, S. L. Keil, S. V. Avakyan (Eds.), Proc. SPIE 4853, 88 (2003).

[6] J.-F. Hochedez, P. Bergonzo, M.-C. Castex, P. Dhez, O. Hainaut, M. Sacchi, J. Alvarez, H. Boyer, A. Deneuville, P. Gibart, B. Guizard, J.-P. Kleider, P. Lemaire, C. Mer, E. Monroy, E. Muñoz, P. Muret, F. Omnes, J. L. Pau, V. Ralchenko, D. Tromson, E. Verwichte, and J.-C. Vial, Diamond UV detectors for future solar physics missions, Diamond and Related Materials 10(3-7), 673 (2001).

[7] S. Koizumi, K. Watanabe, F. Hasegawa, and H. Kanda, Science 292, 1899 (2001).

[8] S. Koizumi, in: C. E. Nebel, J. Ristein (Eds.), Semiconductors and Semimetals 76 - Thin Film Diamond I, Elsevier Academic Press, Amsterdam, 2003, p. 230.

[9] M. Richter, J. Hollandt, U. Kroth, W. Paustian, H. Rabus, R. Thornagel, and G. Ulm, Source and detector calibration in the UV and VUV at BESSY II, Metrologia 40, 107 (2003).

[10] T. Saito, Difference in the photocurrent of semiconductor photodiodes depending on the polarity of current measurement through a contribution from the photoemission current, Metrologia 40, 159 (2003). 